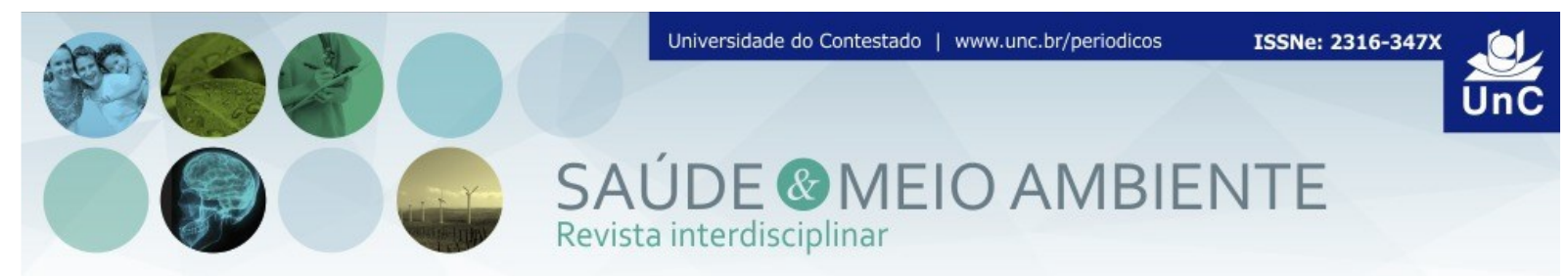

\title{
UTILIZAÇÃO DA CASCA DE OVO DE GALINHA COMO ESTRATÉGIA DE AUMENTO DE CÁLCIO EM ALIMENTOS
}

\section{USE OF CHICKEN EGGSHELL AS A STRATEGY TO INCREASE CALCIUM IN FOOD}

\author{
Luciana Pandolfi ${ }^{1}$ \\ Cátia dos Santos Branco ${ }^{2}$ \\ Elizete Maria Pesamosca Facco ${ }^{3}$ \\ Gabriela Chilanti $^{4}$
}

\begin{abstract}
RESUMO
Introdução: A casca do ovo da galinha é um alimento rico em cálcio, pois contém cerca de $40 \%$ de carbonato de cálcio em sua composição e sua fortificação em alimentos é uma alternativa sustentável para atender as necessidades da população com deficiência desse micronutriente. Objetivo: Desenvolver dois produtos alimentícios (pão e sorvete) acrescidos de $5 \mathrm{~g}$ do pó da casca do ovo para cada 100 $\mathrm{g} / \mathrm{ml}$ de farinha/ou leite usados na formulação, visando um aumento do teor de cálcio e analisar a qualidade microbiológica. Métodos: Foram realizadas análises de composição nutricional, quantificação do teor de cálcio e a qualidade microbiológica (Coliformes termotolerantes, Estafilococos coagulase positiva e Salmonella sp.) da casca do ovo e dos produtos elaborados. Resultados: Os teores de cálcio presentes nos alimentos fortificados variaram de $755 \mathrm{mg}$ a $1,180 \mathrm{mg} / 100 \mathrm{~g}$, valores significativamente mais elevados que as formulações sem a fortificação $(p<0,05)$. Os produtos fortificados tiveram incremento no conteúdo mineral total (cinzas) e redução no teor de açúcar e valor energético. A casca submetida a diferentes métodos de higienização não apresentou contaminação por Coliformes termotolerantes, Estafilococos ou Salmonella $s p$, assim como os produtos fortificados, os quais apresentaram-se dentro dos parâmetros microbiológicos exigidos pela legislação. Conclusão: A fortificação de alimentos com o pó da casca do ovo poderá contribuir para um aumento da ingestão diária de cálcio e prevenção da deficiência desse nutriente, além de ser um alimento seguro para o consumo humano, pois apresenta boa qualidade higiênico-sanitária quando processado de maneira adequada.
\end{abstract}

Palavras-chave: Aproveitamento integral dos alimentos. Alimentos fortificados. Microbiologia de alimentos.

\footnotetext{
${ }^{1}$ Curso de Nutrição. Universidade de Caxias do Sul. Caxias do Sul. Rio Grande do Sul. Brasil. E-mail: Ipandolfi@ucs.br

2Laboratório de Estresse Oxidativo e Antioxidantes. Instituto de Biotecnologia. Universidade de Caxias do Sul. Caxias do Sul. Rio Grande do Sul. Brasil. E-mail: csbranc1@ucs.br

${ }^{3}$ Laboratório de Bromatologia. Universidade de Caxias do Sul. Caxias do Sul. Rio Grande do Sul. Brasil. E-mail: empfacco@ucs.br

${ }^{4}$ Curso de Nutrição. Universidade de Caxias do Sul. Caxias do Sul. Rio Grande do Sul. Brasil. E-mail: gchilant@ucs.br
} 


\begin{abstract}
Introduction: The eggshell of the chicken is a food rich in calcium, as it contains about $40 \%$ of calcium carbonate in its composition and its fortification in food is a sustainable alternative to meet the needs of the population with a deficiency of this micronutrient. Objective: To develop two food products (bread and ice cream) plus $5 \mathrm{~g}$ of eggshell powder for each $100 \mathrm{~g} / \mathrm{ml}$ of flour/or milk used in the formulation, aiming an increase in the calcium, and to analyze the microbiological quality. Methods: Analyses of nutritional composition, quantification of calcium levels, and the microbiological quality (Thermotolerant coliforms, Coagulase-positive Staphylococci, and Salmonella sp.) of eggshell and prepared products were performed. Results: Calcium levels present in fortified foods changed from $755 \mathrm{mg}$ to $1,180 \mathrm{mg} / 100 \mathrm{~g}$, values significantly higher than the formulations without fortification $(p<0.05)$. Fortified products had an increase in total mineral content (ash) and a reduction in sugar content and energy value. The eggshell submitted to different methods of hygiene did not present contamination by Thermotolerant coliforms, Staphylococci, or Salmonella $s p$, as well as the fortified products, which were accorded to what is established by the legislation. Conclusion: The fortification of food with the eggshell powder contributes to an increase in the daily intake of calcium and prevention of the deficiency of this nutrient, besides being a safe food for human consumption, because it presents good hygienic-sanitary quality when processed properly.
\end{abstract}

Keywords: Whole Utilization of Foods. Food Fortified. Food Microbiology.

\title{
INTRODUÇÃO
}

O cálcio é um micronutriente essencial para o corpo humano, sendo importante para o crescimento e manutenção dos ossos e dentes, além de auxiliar na coagulação sanguínea e contração muscular ${ }^{(1)}$. A ingestão deficiente desse micronutriente desde os primeiros anos de vida pode ser um fator de risco para o desenvolvimento de osteoporose na vida adulta, já que afeta a resistência e a qualidade mineral óssea em formação, o que deixa o indivíduo mais suscetível a quedas e fraturas ${ }^{(1,2)}$. Os dados mais recentes disponíveis apontam que, mundialmente, cerca de 8,9 milhões de fraturas ocorrem ao ano em decorrência da osteoporose ${ }^{(1)}$.

A população brasileira apresenta uma ingestão deficiente de cálcio. A ingestão média varia de 300 a 500 mg/dia (2), valores muito abaixo dos recomendados pelas DRIS (Dietary Reference Intakes) que é de 700 a 1,300 mg diários conforme idade e sexo ${ }^{(3)}$.

As principais fontes de cálcio provenientes da dieta são o leite e os laticínios (queijos, iogurtes). O mineral também pode ser encontrado em alguns vegetais (agrião, couve, espinafre, brócolis), cereais (farinha de trigo e de milho) e produtos de origem animal (ovos, sardinhas, camarões e lambari) ${ }^{(1)}$. O cálcio tem sua absorção reduzida com o consumo de alimentos ricos em ácido oxálico, presente no espinafre, batata-doce e feijão, sendo considerado o inibidor mais potente de sua absorção. Já a lactose, presente em leites e derivados, pode aumentar a absorção desse mineral(2). 
De acordo com os dados mais recentes da Associação Brasileira de Proteína Animal (4), o consumo per capita de ovos no Brasil foi de 230 unidades em 2019. Só no estado do Rio Grande do Sul a estimativa de produção reportada pela Associação Gaúcha de Avicultura, é de aproximadamente 3,5 bilhões de unidades, sendo o consumo per capita no estado superior à média nacional (257/habitantes/ano) ${ }^{(5)}$. Com a crescente utilização de ovos pela população e pela indústria de alimentos, o número de cascas de ovos desprezadas vem aumentando. Ao ano, cerca de 6 milhões de toneladas são descartadas nas empresas alimentícias e nas residências no mundo todo, gerando um grande impacto econômico, social e ambiental ${ }^{(6)}$.

Há estudos que comprovam que a casca de ovo de galinha é uma excelente fonte de cálcio ${ }^{(6,7)}$, pois contém cerca de $40 \%$ de carbonato de cálcio em sua composição ${ }^{(8)}$. Sua utilização na fortificação de alimentos poderia se constituir em uma estratégia valiosa para aumentar a ingestão diária de cálcio e atender as necessidades da população que apresenta deficiência desse micronutriente, além de contribuir para a redução do grande número de resíduos gerados nas indústrias de ovos ${ }^{(9)}$.

Uma preocupação que existe nesse sentido é em relação a qualidade microbiológica dessa matéria-prima, uma vez que o consumo de ovos in natura ou em preparações tem sido associados com intoxicações alimentares por estafilococos e/ou Salmonella(6). Assim, a higienização no preparo é de vital importância para a inocuidade das cascas e a segurança dos consumidores.

Nesse sentido, o presente estudo teve por objetivo desenvolver dois produtos alimentícios (pão integral e sorvete) fortificados com o pó da casca de ovo de galinha, como proposta para promover o aproveitamento integral do alimento, reduzir o desperdício e os impactos ambientais visando um potencial benefício à saúde humana ao aumentar o teor de cálcio na composição do produto, Paralelamente, a segurança microbiológica das cascas de ovo e dos produtos elaborados foi também avaliada.

\section{MATERIAIS E MÉTODOS}

\section{MATÉRIA-PRIMA E PREPARO}

Para a obtenção das cascas de ovos de galinha, foi adquirida uma dúzia de ovos em mercado local da cidade de Caxias do Sul (RS), esta foi acondicionada sob refrigeração até o processamento e obtenção do pó da casca do ovo. Para aproveitar integralmente o alimento, as gemas e claras foram destinadas para a elaboração do sorvete.

As cascas foram submetidas a dois tipos de tratamentos visando investigar os efeitos da fervura na presença ou ausência do hipoclorito, usado como sanitizante na desinfecção. No tratamento 1 foi realizada a lavagem em água corrente, imersão em hipoclorito de sódio por 10 minutos e fervura durante 10 minutos. Após esse procedimento, as cascas foram secas em forno elétrico (FISCHER, modelo 9741- 
12875, SC-Brasil) a $150{ }^{\circ} \mathrm{C}$ por 40 minutos, trituradas em processador doméstico (ARNO, Optimix plus, SP-Brasil), peneiradas para a obtenção de um pó e armazenadas em recipientes escuros, de acordo com a metodologia empregada no estudo de Naves e colaboradores ${ }^{(10)}$. No tratamento 2, as cascas não foram submetidas a imersão em hipoclorito de sódio e fervura em água durante 10 minutos, sendo que as demais etapas foram realizadas.

\section{ELABORAÇÕES DAS FORMULAÇÕES FORTIFICADAS}

Foram fortificados com o pó da casca do ovo dois produtos de fácil acesso e preparo (pão integral e sorvete). Foram adicionadas 5 gramas do pó da casca do ovo para cada 100 gramas de farinha utilizado no preparo do pão e 5 gramas para cada $100 \mathrm{ml}$ de leite utilizados na formulação do sorvete. As quantidades de cascas a serem adicionadas foram definidas a partir de testes prévios realizados durante a pesquisa. Para definição da quantidade de farinha utilizada, foram adicionadas nos produtos 3 concentrações de pó da casca do ovo $(2,5 \mathrm{~g}, 5 \mathrm{~g}$ e $10 \mathrm{~g})$, os produtos foram avaliados sensorialmente pelos pesquisadores, e optou-se pela quantidade intermediaria, pois $5 \mathrm{~g}$ não alterou as características iniciais do produto.

Os produtos desenvolvidos foram elaborados de maneira manual, a fim de simular uma produção caseira. Na Figura 1 e 2 estão descritas as etapas de processamento da fabricação do pão e sorvete.

Figura 1 - Etapas da fabricação do pão integral.

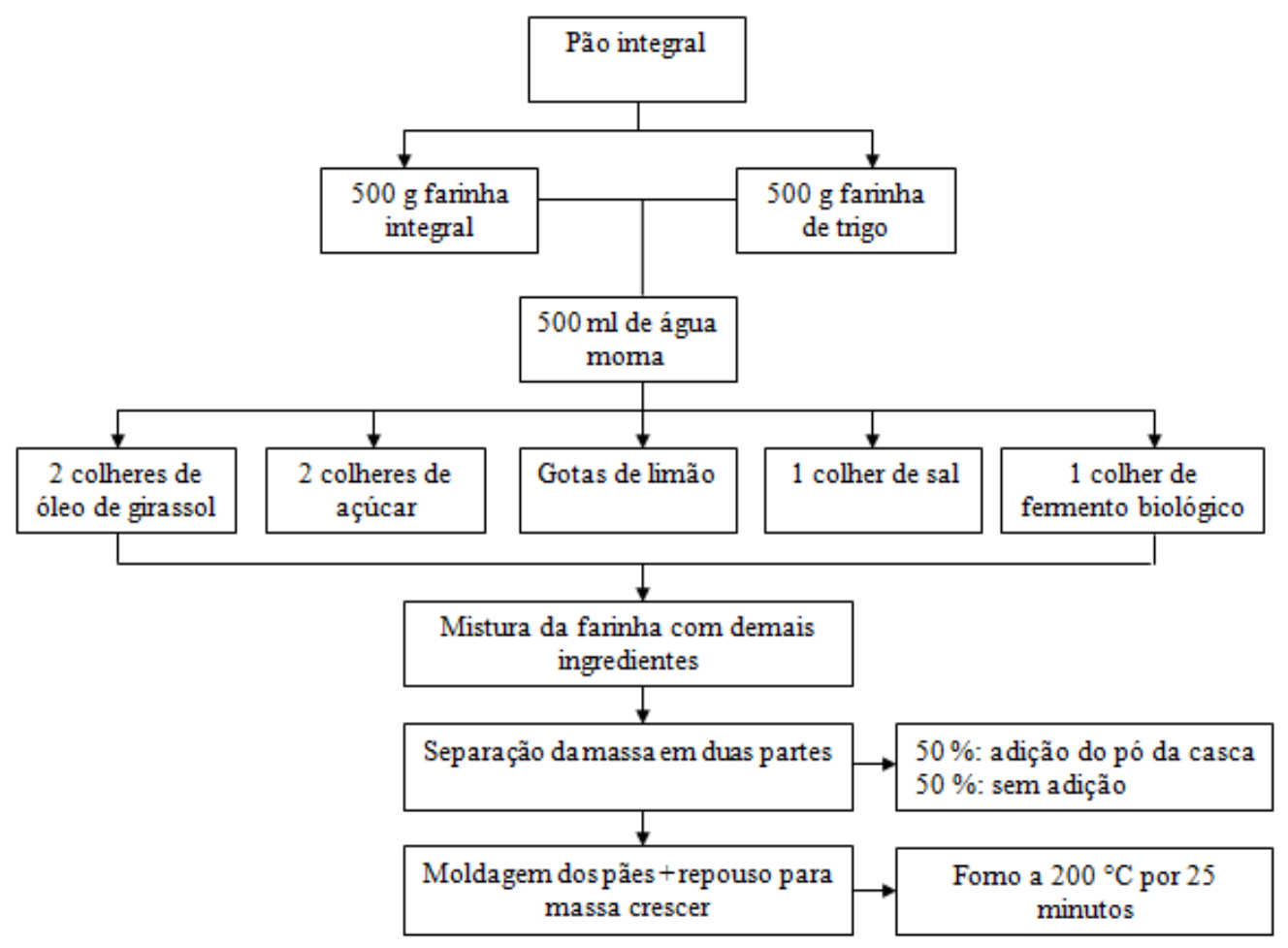


Figura 2 - Etapas da fabricação do sorvete.

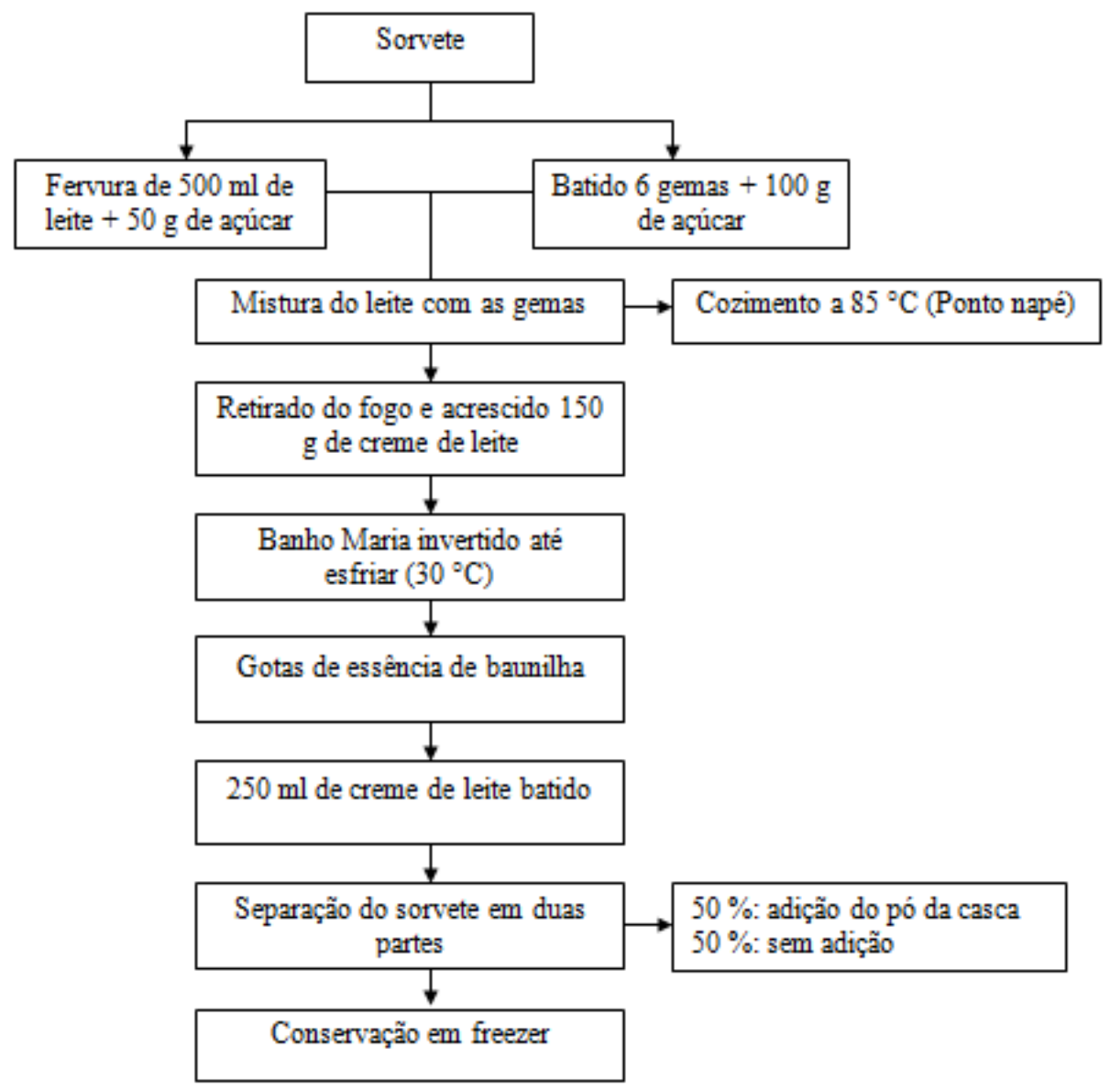

\section{ANÁLISES MICROBIOLÓGICAS}

As amostras foram analisadas quantitativamente quanto a presença de Coliformes termotolerantes, Estafilococos coagulase positiva e Salmonella sp. nos alimentos fortificados com o pó da casca do ovo e nos alimentos sem a fortificação, bem como na casca do ovo com o processamento adequado de higienização em hipoclorito de sódio descrita no tratamento 1 e da casca sem a higienização adequada citada no tratamento 2 . As análises e interpretação dos resultados foram realizadas de acordo com os critérios microbiológicos estabelecidos pela Legislação Brasileira(11).

\section{DETERMINAÇÃO DA COMPOSIÇÃO CENTESIMAL DOS ALIMENTOS ELABORADOS}

A umidade foi realizada pelo método de secagem em estufa a $105^{\circ} \mathrm{C}$ até peso constante. A determinação de cinzas foi realizada pelo método da incineração a 600 ${ }^{\circ} \mathrm{C}$ em mufla. As proteínas foram determinadas pelo método de micro Kjeldhal, com 
fator de conversão 6,25. Os lipídeos foram obtidos pela extração com a combinação de solventes à frio e quantificados por gravimetria. Para a determinação de carboidratos calculou-se a diferença entre $100 \mathrm{~g}$ do alimento e a soma total dos valores encontrados para umidade, cinzas, proteína e lipídeos. O cálcio foi determinado pelo método de espectrometria de absorção atômica com chama em uma amostra representativa do alimento. Todas as análises foram realizadas em duplicata e de acordo com os métodos descritos pela Association of Official Analytical Chemists ${ }^{(12)}$ e Instituto Adolfo Lutz ${ }^{(13)}$.

O valor calórico total dos alimentos foi estimado considerando-se os fatores de conversão de Atwater de $4 \mathrm{kcal} / \mathrm{g}$ de carboidrato, $4 \mathrm{kcal} / \mathrm{g}$ de proteína, e $9 \mathrm{kcal} / \mathrm{g}$ de lipídio. Em seguida os dados de energia foram convertidos para Joule (Sistema Internacional de Medidas), utilizando o fator de 4,1868 kJ para $1 \mathrm{kcal}$.

\section{ANÁLISE ESTATÍSTICA}

Os resultados foram expressos em média \pm desvio padrão. Os dados foram analisados por variância (one-way ANOVA) e pós-teste de Tukey, considerando nível de probabilidade inferior a $5 \%(p<0,05)$, utilizando o software Prism GraphPad $\AA$ (versão 5.0.1.334).

\section{RESULTADOS}

Os resultados da composição centesimal e teores de cálcio dos produtos (pão e sorvete) com e sem a fortificação estão descritos nas Tabelas 1 e 2.

Tabela 1 - Caracterização físico-química e teor de cálcio em $100 \mathrm{~g}$ de pão integral com e sem a adição do pó da casca do ovo.

\begin{tabular}{lcc}
\hline & Pão não fortificado & Pão fortificado \\
\cline { 2 - 3 } Umidade & $41,65 \pm 0,05^{\mathrm{a}}$ & $40,24 \pm 0,05^{\mathrm{b}}$ \\
Cinzas & $2,41 \pm 0,01^{\mathrm{a}}$ & $5,67 \pm 0,02^{\mathrm{b}}$ \\
Proteína & $9,17 \pm 0,35^{\mathrm{a}}$ & $9,04 \pm 0,10^{\mathrm{a}}$ \\
Lipídios & $2,05 \pm 0,00^{\mathrm{a}}$ & $1,79 \pm 0,00^{\mathrm{b}}$ \\
Carboidrato & $44,72 \pm 0,40^{\mathrm{a}}$ & $43,26 \pm 0,05^{\mathrm{b}}$ \\
Energia (kcal) & $234,0 \pm 0,22^{\mathrm{a}}$ & $225,32 \pm 0,36^{\mathrm{b}}$ \\
Energia $(\mathrm{kJ})$ & $979,71 \pm 0,93^{\mathrm{a}}$ & $943,38 \pm 1,52^{\mathrm{b}}$ \\
\hline Cálcio $(\mathrm{mg})$ & $18,50 \pm 0,50^{\mathrm{a}}$ & $755,00 \pm 15,00^{\mathrm{b}}$ \\
\hline
\end{tabular}

Os valores apresentados são resultantes da média de duas repetições. Em cada linha, letras iguais não diferem significativamente $(p<0,05)$. 
Tabela 2 - Caracterização físico-química e teor de cálcio em $100 \mathrm{~g}$ de sorvete com e sem a adição do pó da casca do ovo.

\begin{tabular}{lcc}
\hline & Sorvete não fortificado & Sorvete fortificado \\
\cline { 2 - 3 } Umidade & $61,57 \pm 0,62^{\mathrm{a}}$ & $63,20 \pm 0,15^{\mathrm{b}}$ \\
Cinzas & $0,78 \pm 0,01^{\mathrm{a}}$ & $4,23 \pm 0,09^{\mathrm{b}}$ \\
Proteína & $3,40 \pm 1,10^{\mathrm{a}}$ & $3,71 \pm 0,03^{\mathrm{a}}$ \\
Lipídios & $8,82 \pm 0,00^{\mathrm{a}}$ & $9,98 \pm 0,00^{\mathrm{b}}$ \\
Carboidrato & $25,43 \pm 0,13^{\mathrm{a}}$ & $18,89 \pm 0,04^{\mathrm{b}}$ \\
Energia (kcal) & $194,69 \pm 0,67^{\mathrm{a}}$ & $180,22 \pm 0,26^{\mathrm{b}}$ \\
Energia (kJ) & $815,11 \pm 2,82^{\mathrm{a}}$ & $753,05 \pm 1,02^{\mathrm{b}}$ \\
\hline Cálcio (mg) & $71,50 \pm 0,50^{\mathrm{a}}$ & $1,180 \pm 2,00^{\mathrm{b}}$ \\
\hline
\end{tabular}

Os valores apresentados são resultantes da média de duas repetições. Em cada linha, letras iguais não diferem significativamente $(p<0,05)$.

Em relação a composição centesimal dos pães, pode-se observar que houve aumento significativo de cerca de 2,5 vezes no teor de cinzas (minerais totais) no pão fortificado. Adicionalmente, o teor de cálcio presente no mesmo foi cerca de 40 vezes superior ao pão sem a fortificação. Nos demais parâmetros, esse alimento exibiu valores diminuídos para teor de umidade, lipídeos, carboidratos e valor energético (redução de 3, 13, 3 e 4 \%, respectivamente) em relação ao pão sem a fortificação (Tabela 1).

Os sorvetes elaborados com as cascas de ovo apresentaram um incremento de cerca de 5 vezes no teor total de cinzas e cerca de 16 vezes na quantidade de cálcio. Houve aumento também nos parâmetros de umidade (cerca de $3 \%$ ) e $12 \%$ nos teores de lipídeos. Semelhante ao observado para os pães fortificados, houve redução de $25 \%$ no teor de carboidratos, o que resultou em uma redução de aproximadamente $8 \%$ no valor energético o total do sorvete (Tabela 2). Para ambos os produtos elaborados, o conteúdo de proteína foi o único parâmetro que não apresentou alteração estatisticamente significativa antes e após a fortificação.

Na Tabela 3 estão descritos os valores de cálcio encontrados nos alimentos fortificados e não fortificados e sua comparação com as recomendações diárias de cálcio para crianças, adolescentes, adultos e idosos.

Tabela 3 - Comparação da recomendação de ingestão dietética de cálcio conforme faixa etária com o teor de cálcio presentes em $100 \mathrm{~g}$ de pão e sorvete fortificados com o pó da casca do ovo e os sem a fortificação.

\begin{tabular}{|c|c|c|c|c|c|c|}
\hline \multicolumn{7}{|c|}{ Porcentagem (\%) em relação à IDR* } \\
\hline \multirow[b]{3}{*}{ PÃO } & & $\begin{array}{c}\text { Cálcio } \\
\text { mg/100g }\end{array}$ & $\begin{array}{c}\text { Criança } \\
\text { (4 a } 8 \\
\text { anos) }\end{array}$ & $\begin{array}{c}\text { Adolescente } \\
\text { (9 a } 18 \\
\text { anos) }\end{array}$ & $\begin{array}{l}\text { Adulto } \\
\text { (19 a 50 } \\
\text { anos) }\end{array}$ & $\begin{array}{l}\text { Idoso } \\
\text { (>70 } \\
\text { anos) }\end{array}$ \\
\hline & Fortificado & 755 & 76 & 58 & 76 & 76 \\
\hline & Não fortificado & 18,5 & 2 & 1 & 2 & 2 \\
\hline \multirow{3}{*}{ SORVETE } & Fortificado & 1,180 & 100 & 91 & 100 & 100 \\
\hline & Não fortificado & 71,5 & 7 & 6 & 7 & 7 \\
\hline & IDR (mg/dia) & - & 1.000 & 1.300 & 1.000 & 1.000 \\
\hline
\end{tabular}

*IDR: ingestão dietética recomendada. 
É possível observar que o consumo de 100 gramas do pão fortificado com o pó da casca do ovo atinge $76 \%$ da recomendação diária para crianças, adultos e idosos e $58 \%$ para adolescentes. Já o sorvete fortificado atinge $100 \%$ da recomendação diária para crianças, adultos e idosos e $91 \%$ para adolescentes, enquanto que o sem a fortificação atinge apenas $7 \%$ e $6 \%$ respectivamente.

Os resultados da análise microbiológica estão descritos na Tabela 4. As cascas de ovos submetidas aos diferentes processos de higienização (Tratamentos 1 e 2) não apresentaram contaminação por coliformes termotolerantes, estafilococos coagulase positiva e Salmonella sp.

O pão desenvolvido no estudo também não apresentou contaminação microbiológica (Estafilococos coagulase-positiva: $<1 \times 10^{1} \mathrm{UFC} / \mathrm{g}$ e Coliformes termotolerantes: $\left.<1 \times 10^{1} \mathrm{NMP} / \mathrm{g}\right)$. Já o sorvete apresentou contaminação por Coliformes termotolerantes $\left(2,3 \times 10^{1} \mathrm{NMP} / \mathrm{g}\right)$ e Estafilococos (4 a $\left.5 \times 10^{1} \mathrm{UFC} / \mathrm{g}\right)$, embora dentro dos padrões exigidos pela legislação.

Tabela 4 - Análise microbiológica dos alimentos formulados com e sem a adição do pó da casca do ovo e da casca submetida a diferentes processos de higienização.

\section{Microrganismos}

\begin{tabular}{|c|c|c|c|}
\hline & $\begin{array}{l}\text { Salmonella sp. } \\
\text { (em 25g de } \\
\text { amostra) }\end{array}$ & $\begin{array}{c}\text { Estafilococos } \\
\text { coagulase positiva } \\
\text { (UFC/g) }^{1}\end{array}$ & $\begin{array}{c}\text { Coliformes } \\
\text { termotolerantes } \\
(\mathrm{NMP} / \mathrm{g})^{2}\end{array}$ \\
\hline Casca do ovo $1^{3}$ & Ausência & $<1 \times 10^{1}$ & $<1 \times 10^{1}$ \\
\hline Casca do ovo $2^{4}$ & Ausência & $<1 \times 10^{1}$ & $<1 \times 10^{1}$ \\
\hline Pão não fortificado & Ausência & $<1 \times 10^{1}$ & $<1 \times 10^{1}$ \\
\hline Pão fortificado & Ausência & $<1 \times 10^{1}$ & $<1 \times 10^{1}$ \\
\hline Sorvete não fortificado & Ausência & $4 \times 10^{1}$ & $2,3 \times 10^{1}$ \\
\hline Sorvete fortificado & Ausência & $5 \times 10^{1}$ & $2,3 \times 10^{1}$ \\
\hline Padrão microbiológico & Ausência & $5 \times 10^{2}$ & $5 \times 10^{1}$ \\
\hline \multicolumn{4}{|c|}{$\begin{array}{l}\text { 1_UFC/g: unidade formadora de colônia por grama da amostra. } \\
\text { 2- NMP/g: número mais provável por grama da amostra. } \\
\text { 3-Tratamento 1: higienização da casca do ovo com a imersão em hipoclorito de sódio por } 10 \text { minutos } \\
\text { e fervura em água durante } 10 \text { min. } \\
\text { 4- Tratamento 2: higienização da casca do ovo em água corrente, sem imersão em hipoclorito de } \\
\text { sódio e fervura. }\end{array}$} \\
\hline
\end{tabular}

\section{DISCUSSÃO}

Nesse estudo, foram produzidos dois alimentos com emprego de diferentes processamentos térmicos, pães e sorvetes. A fortificação dos pães e sorvetes com o pó das cascas do ovo de galinha não apenas aumentou significativamente os teores de cálcio, como o teor total de cinzas. Esse achado é importante, já que as cinzas representam o conteúdo total de minerais resultantes da queima da matéria orgânica de um alimento ${ }^{(16)}$. Esse aumento de cinzas nos produtos fortificados se deu pela grande quantidade de minerais presentes na casca do ovo, que além do cálcio contém magnésio, estrôncio, ferro, selênio e outros, embora em menores quantidades ${ }^{(6)}$. No 
estudo de Oliveira e colaboradores $^{(17)}$ que objetivou a elaboração de pão suplementado com o extrato de citrato de cálcio obtido a partir de cascas de ovos de galinha brancas, observou-se maiores quantidades de cinzas no pão suplementado com a casca quando comparado ao pão sem suplementação, corroborando os achados do presente estudo.

Foram encontrados teores de umidade, lipídeos e calorias menores nos alimentos fortificados, bem como carboidratos por ser quantificado por diferença. Esses resultados podem ser explicados pelo fato de que as cinzas presentes em uma maior quantidade na amostra reduzem as concentrações desses nutrientes ${ }^{(18)}$. Em relação ao teor proteico dos produtos elaborados, não foram encontradas diferenças estatísticas quanto comparados entre si. Esses teores podem ser explicados pela presença de leite e creme de leite usado na formulação do sorvete e farinha de trigo e farinha integral utilizados no preparo do pão. O creme de leite apresenta $1,5 \mathrm{~g} / 100 \mathrm{~g}$ de proteína ${ }^{(18)}$ e o leite integral $3 \mathrm{~g} / 100 \mathrm{~g}^{(19)}$. Já a farinha de trigo apresenta 9,8 $\mathrm{g} / 100 \mathrm{~g}$ de proteína ${ }^{(18)}$. Em vista disso, o pão integral apresentou um teor maior de proteína quando comparado ao sorvete. Esses resultados corroboram os dados demonstrados previamente por Naves e colaboradores ${ }^{(7)}$.

Especificamente em relação ao cálcio, o incremento do teor no pão e no sorvete ocorreu devido à casca do ovo ser composta de aproximadamente $40 \%$ de carbonato de cálcio ${ }^{(8)}$. Similarmente, no estudo de Naves e colaboradores ${ }^{(7)}$, foi reportado teores de cálcio entre 111,5 e 506,4 mg/100g em diferentes produtos (arroz cozido, arroz doce, beiju com margarina, biscoito de polvilho, biscoito frito doce, biscoito frito salgado, bolinho de chuva, bolo de fubá de milho, bolo simples, farofa de ovo e banana) após fortificação.

De acordo com a Agência Nacional de Vigilância Sanitária (ANVISA), alimento "fonte" de vitamina e minerais é aquele com no mínimo $15 \%$ da ingestão diária recomendada por $100 \mathrm{~g}$ de alimento ou por porção, e o alimento com "alto conteúdo" de vitamina e minerais é aquele com no mínimo $30 \%$ da ingestão diária recomendada por $100 \mathrm{~g}$ de alimento ou por porção ${ }^{(20)}$. Diante disso, pode-se afirmar que o pão e o sorvete fortificados e elaborados no presente estudo são classificados como alimentos com "alto conteúdo" de cálcio.

O consumo de alimentos fortificados pode atingir até $100 \%$ da recomendação diária de cálcio para crianças, adolescentes, adultos e idosos. Os grupos mais vulneráveis que necessitam de uma maior ingestão podem se beneficiar destes alimentos. Ao comparar a Tabela $3 \mathrm{com}$ os principais alimentos fontes de cálcio podemos observar que o consumo de $100 \mathrm{ml}$ de iogurte natural contém $143 \mathrm{mg}$ de cálcio, representando $14 \%$ da recomendação diária para crianças, adultos e idosos, já o consumo de $100 \mathrm{ml}$ de leite de vaca integral contém $123 \mathrm{mg}$ de cálcio e representa $9 \%$ da recomendação diária para adolescentes. A ingestão de $50 \mathrm{~g}$ de queijo parmesão contém $496 \mathrm{mg}$ de cálcio representando 38\% da recomendação diária para adolescentes ${ }^{(18)}$. Para a população que apresenta uma baixa ingestão de alimentos fontes, a utilização de alimentos fortificados pode ser uma boa alternativa para atingir a necessidade diária recomendada. 
É de extrema importância a avaliação microbiológica dos alimentos para a garantia de um produto de qualidade e seguro ao consumo humano, evitando o surgimento de doenças transmitidas por alimentos (DTAs) e dos possíveis danos à saúde do consumidor ${ }^{(21)}$. No presente estudo foi demonstrado que as cascas de ovos submetidas aos diferentes processos de higienização (aplicação ou não de imersão em hipoclorito de sódio e fervura) não apresentaram contaminação por Coliformes termotolerantes, Estafilococos coagulase positiva ou Salmonella sp., indicando uma boa qualidade higiênico-sanitária. Os mesmos achados foram encontrados no estudo de Naves e colaboradores ${ }^{(10)}$ e Milbradt e colaboradores ${ }^{(6)}$ o que corrobora a eficácia dos processos de higienização aqui aplicados. Em relação aos produtos elaborados, observou-se que o pão não apresentou contaminação microbiológica (Estafilococos: $<1 \times 10^{1} \mathrm{UFC} / \mathrm{g}$ e Coliformes termotolerantes: $\left.<1 \times 10^{1} \mathrm{NMP} / \mathrm{g}\right)$. Já o sorvete apresentou contaminação por Coliformes termotolerantes $\left(2,3 \times 10^{1} \mathrm{NMP} / \mathrm{g}\right)$ e Estafilococos (4 a $5 \times 10^{1} \mathrm{UFC} / \mathrm{g}$ ), com provável contaminação da matéria-prima utilizada para a formulação e pelo fato desse alimento não se submeter a temperaturas elevadas, diferentemente do pão. Apesar disso, os resultados apresentaram-se dentro dos parâmetros microbiológicos estabelecidos pela Legislação ${ }^{(11)}$.

O presente estudo demonstrou que a casca do ovo de galinha, quando submetida à higienização adequada, torna-se segura ao consumo humano e contribui para o aumento dos níveis de cálcio, acarretando em benefícios à saúde humana, além de estimular o aproveitamento do alimento como um todo. Essa é uma estratégia que deve ser incentivada, visto que o desperdício de alimentos é um problema que acontece em nível mundial.

Segundo os dados do Banco de Alimentos ${ }^{(14)}$, mais de 40 mil toneladas são desperdiçadas todos os dias no Brasil gerando um grande impacto econômico e ambiental. $\mathrm{O}$ aproveitamento integral dos alimentos consiste em pequenas mudanças no cotidiano para minimizar ao máximo possível o desperdício de alimentos, reduzir a geração de lixo, reduzir gastos com alimentação, além de obter os nutrientes que seriam desprezados ${ }^{(15)}$.

O presente estudo demonstrou a viabilidade de utilizar, de forma segura, cascas de ovos em diferentes matrizes alimentícias, envolvendo extremos de temperatura. Esses achados são importantes, pois reforçam a utilização desse subproduto como fonte de cálcio para a população(22). Futuros estudos são necessários, no entanto, para avaliar sensorialmente os produtos em diferentes públicos, assim como o perfil de biodisponibilidade do cálcio in vivo.

\section{CONCLUSÃO}

O presente estudo evidenciou os benefícios decorrentes da utilização do pó da casca do ovo na fortificação de alimentos (pão e sorvete) como uma proposta viável e de baixo custo. Seu uso em preparações de fácil acesso à população pode resultar em um aumento da ingestão diária de cálcio e consequente prevenção de doenças relacionadas à sua carência, como a osteoporose. Ademais, a casca do ovo de galinha submetida aos diferentes processos de higienização pode ser considerada um 
alimento seguro para o consumo humano, pois não apresentou contaminação microbiológica, em especial ao gênero Salmonella, indicando uma boa qualidade higiênico-sanitária. Por fim, os dados obtidos nesse trabalho reiteram a importância do aproveitamento integral dos alimentos, reduzindo o desperdício e minimizando impactos ambientais.

\section{REFERÊNCIAS}

1. Martini LA, Peters BSE. Cálcio e vitamina D [Internet]. Barueri: Manole; 2017. Available from: https://www.piattoveg.info/calcio.html

2. Cozzolino SMF. Biodisponibilidade de nutrientes. 5th ed. Barueri: Manole; 2016.

3. Ross C, Taylor CL, Yaktine AL, Valle HB Del. Dietary Reference Intakes for Calcium and Vitamin D. Washington DC: National Academies Press; 2011.

4. ABPA. Associação Brasileira de Proteína Animal [Internet]. 2021 [cited 2021 Feb 12]. Available from: https://abpa-br.org/mercados/

5. ASGAV. Associação Gaúcha de Avicultura [Internet]. 2021 [cited 2021 Feb 12]. Available from: https://www.asgav.com.br/index.php/estatisticas

6. Milbradt BG, Müller ALH, da Silva JS, Lunardi JR, Milani LIG, De Moraes Flores ÉM, et al. Casca de ovo como fonte de cálcio para humanos: Composição mineral e análise microbiológica. Cienc Rural. 2015;45(3):560-6.

7. Naves MMV, Fernandes DC, Prado CMM, Teixeira LSM. Fortificação de alimentos com o pó da casca de ovo como fonte de cálcio. Cienc e Tecnol Aliment. 2007;27(1):99-103.

8. Peres AP, Waszczynskyj N. Farinha de casca de ovo : determinação do teor de cálcio biodisponível. Visão Acadêmica, Curitiba. 2010;11(1):74-80.

9. Dallacorte C, Behling SM, Quadros CS de. Implantação de uma indústria de farinha de casca de ovo: um estudo da viabilidade econômica. Rev Tecnológica [Internet]. 2017;6(1):83-103. Available from:

https://uceff.edu.br/revista/index.php/revista/article/view/236

10. Naves MMV, Prado CMM, Fernandes DC, Serafini ÁB. Avaliação microbiológica do pó da casca de ovo e otimização da técnica de elaboração do produto.

Pesqui Agropecuária Trop. 2007;37(2):113-8.

11. BRASIL. RESOLUÇÃO ANVISA - RDC No 12, de 2 de janeiro de 2001 [Internet]. 2001 [cited 2018 Mar 13]. p. 1-40. Available from:

file://C:/Users/catia/Downloads/Resolucao RDC ANVISA 12 - 2001 -Padroes

Microbiologicos de Alimentos.pdf 
12. AOAC. The Official Methods of Analysis of AOAC International. 20th ed. George W. Latimer, editor. ROCKVILLE: AOAC INTERNATIONAL; 2016.

13. IAL. Métodos Físico-Químicos para Análise de Alimentos. $4^{a}$. Métodos físicosquimicos para análise de Alimentos. São Paulo: Instituto Adolfo Lutz; 2008.

14. Banco de Alimentos [Internet]. 2021 [cited 2021 Feb 12]. Available from: https://bancodealimentos.org.br/

15. FAO. Food and Agriculture Organization of the United Nations [Internet]. 2021 [cited 2021 Feb 4]. Available from: http://www.fao.org/food-loss-and-foodwaste/es/

16. Krumreich FD, Sousa CT, Corrêa APA, Krolow CR, Zambiazi RC. Teor de cinzas em acessos de abóbora (Curcubita máxima L.) do Rio Grande do Sul. VIII Simp Aliment [Internet]. 2013;8:1-4. Available from:

https://ainfo.cnptia.embrapa.br/digital/bitstream/item/94505/1/cinzas-emaboboras.pdf

17. Oliveira MA de, Ferreira CR, Júnior SR, Saraiva3 SH. Desenvolvimento de uma formulação de pão suplementado com cálcio. Enciclopédia Biosf [Internet]. 2011;7(13):1419-26. Available from: http://www.elsevier.com/locate/scp

18. TACO. Tabela Brasileira de Composição de Alimentos [Internet]. Campinas: NEPA- UNICAMP; 2011. 161 p. Available from:

http://www.nepa.unicamp.br/taco/contar/taco_4_edicao_ampliada_e_revisada.pd f?arquivo=taco_4_versao_ampliada_e_revisada.pdf

19. Philippi ST. Tabela de composição de alimentos : suporte para decisão nutricional. 5th ed. Baruer: Manole; 2016.

20. BRASIL. RESOLUÇÃO ANVISA - RDC Nº 54 , de 12 de novembro de 2012 [Internet]. 2012 [cited 2018 May 11]. p. 1-5. Available from: http://bvsms.saude.gov.br/bvs/saudelegis/anvisa/2012/rdc0054_12_11_2012.htm I

21. Germano PML, Germano MIS. Higiene e vigilância sanitária de alimentos. 6th ed. Barueri: Manole; 2019.

22. Vilar JDS, Oliveira Sabaa-Srur AU, Ruy GM. Composição química da casca de ovo de galinha em pó. Bol Cent Pesqui Process Aliment. 2010;28(2):247-54.

Artigo recebido em: 17/02/2021

Artigo aprovado em: 01/03/2022

Artigo publicado em: 04/03/2022 